1 The HCT Index: A Typology and Index of Health Conspiracy Theories

2 with examples of use

4 Joseph M. Stubbersfield ${ }^{1}$, Tom Widger ${ }^{1}$, Andrew J. Russell ${ }^{1}$, and Jamshid J. Tehrani ${ }^{1}$ 5

$6{ }^{1}$ Durham University, Department of Anthropology, Dawson Building, South Road, Durham, 7 DH1 3LE

\title{
Abstract
}

Conspiracy theories about secret agendas behind vaccination programmes, the side effects of medical treatments, and cover-ups by the government or pharmaceutical industry are prevalent in many countries, and can have highly detrimental and far-reaching effects on people's wellbeing. For, research and policy-making in public health, it is therefore vital to understand the nature, construction and dissemination of these health conspiracy theories (HCTs). Inspired the influential ATU index of folktale types, this paper presents a typology and example index of international HCTs to be used as a tool to enable researchers to identify and categorise HCTs they come across, and to provide a pool of examples of HCTs which could be used in various fields of research. Also presented are two studies which used the HCT Index as a source of material. The first, a survey of HCT exposure and belief in the UK found that both familiarity and belief were high: $97 \%$ of Britons are familiar (having heard the same or similar before) with at least one HCT and $49 \%$ of Britons believed that at least one HCT was likely to be true. Demographic influences are also discussed. The second study, a focus group discussion health rumours in rural Sri Lanka, found concerns over threats to fertility as well as how to verify information that falls outside of typical experience. 
The dissemination of misinformation, such as "Fake News" or conspiracy theories, is a serious global issue in contemporary culture. This issue can have particularly dire consequences when related to health. In South Africa in the 1990s, for example, misguided claims about the origins and best treatments for HIV/AIDS led to the premature deaths of an estimated 330,000 people. More recently Europe saw record high rates of measles cases in 2018 (WHO, 2018), with high profile medical professionals warning the public against misinformation disseminated on social media (Roxby, 2018). Conspiracy theories about secret agendas behind vaccination programmes, the side effects of medical treatments, and cover-ups by the government or pharmaceutical industry are prevalent in many countries, and can have highly detrimental and far-reaching effects on people's wellbeing. Attitudes towards vaccines have become the primary barrier to vaccination, over physical access (CDC, 2013), and research demonstrates that belief in health conspiracy theories (HCTs) is linked to reduced contraceptive use (Bogart \& Thorburn, 2005), reduced intention to vaccinate (Jolley \& Douglas, 2015) and avoidance of mainstream medicine (Oliver \& Wood, 2014). For research and policy-making in public health, it is therefore vital to understand the nature, construction and dissemination of HCTs. A key tool in this endeavour is a typology of HCTs to enable researchers to identify and categorise HCTs they come across, and to provide an international pool of examples of HCTs which could be used in various fields of research.

HCTs can be considered, alongside other urban legends and other conspiracy theories, as a form of contemporary folklore. These are distinguished from more traditional folklore, such fairy tales, by their contemporary or post-industrial setting, but nonetheless resemble traditional folklore in many aspects. The study of traditional folklore has been advanced by the use of the Aarne-Thompson-Uther (ATU) classification system and motif index, named after its authors Antti Aarne, Stith Thompson, and Hans-Jörg Uther. The ATU system is used in folkloristics and other fields examining folklore to organise, classify and analyse traditional folklore narratives through the use of numbered tale types. Each folktale is given a generic title and identifying number and related folktales are grouped by type. For example tales 1-299 are classified as 'Animal Tales', while 300-749 are 'Tales of Magic'. These are further divided by type, with 300-399 all featuring 'Supernatural Adversaries'. Ultimately each folktale type is given its own identifying number, generic title and generic plot 
description with other tale types it may include listed. Variants of the tale type are listed and examples of the tale type are given. For example the entry for Little Red Riding Hood is:

\section{3: Little Red Riding Hood}

The wolf or other monster devours human beings until all of them are rescued alive from his belly. Cf. Types 123, 2027, 2028.

I. Wolf's Feast. (a) By masking as mother or grandmother the wolf deceives and devours (b) a little girl (Red Riding Hood)...

Variants

333A: Cattarinetta

Little girl goes to wolf (ogre) to borrow a pan in which to prepare pancake. On the way back to return pan with fritters, she eats them and substitutes horse dung (dirt). She then hides from the wolf. He unties the horse and eats her. (Sometimes she...

333B: The cannibal godfather (godmother)

The little girl disregards the warning of friendly animals and visits the godfather (godmother). She is devoured.

Examples

$\begin{array}{llllll}\text { ATU } & \text { Author } & \text { Year } & \text { County } & \text { Language } & \text { Title } \\ 333 & \text { Charles Perrault/ } & 1889 & \text { France } & \text { English } & \text { Little Red Riding } \\ & \text { Andrew Lang } & & & & \text { Hood }\end{array}$

89 In addition to the classification of tale types the ATU system also provides an index of motifs. These are organised by a general topic, for example category E is 'the Dead'. These are further divided into more specific categories, such as E431, 'Precautions at funeral against revenant' and subcategories, such as E431.13, 'Corpse burned to prevent return'.

Dundes (1997) describes the ATU indices as the "most valuable tools in the professional folklorist's arsenal of aids for analysis", and their utility is demonstrated by their practical use in both qualitative and quantitative research. A recent example of its use in quantitative research is Bortolini et al (2017) who used folktales sourced from the ATU index in 
conjuction with worldwide whole-genome sequences to examine the transmission mechanisms of traditional folktales in Eurasia and Africa.

Inspired by the current ATU system (Uther, 2004) and Brunvand's (1963) type index of "Shaggy Dog Stories", this paper presents a typology and categorisation system, including motifs, for international HCTs, the HCT Index. With the intention that this index can be used in future research examining HCTs in broad range of fields, as the ATU system has been used in the research of traditional folklore. We also present two studies that used the index in some form as illustrative examples of its utility.

\section{Method and construction}

HCTs were collected primarily from three sources: peer-reviewed academic literature on the subject of conspiracy theories, news and magazine journalism and conspiracy theorist websites. Initial collection was from academic literature and news journalism identified in a systematic literature review performed as part of the activities of the Wolfson Research Institute for Health and Wellbeing Conspiracy Theories in Health Special Interest Group at Durham University. Further, we conducted a survey of known health-related or general conspiracy theorist websites, such as naturalnews.com, and books, such as David Icke's The biggest secret: The book that will change the world. In addition, we conducted web searches based around known HCTs. See Appendix A for the full list of sources. HCTs were added to the index if they met the criteria of the working definition of an HCT provided below:

A belief that a either a group of individuals or an organisation is deliberately and secretly:

a) Causing harm to the health of the general public or a specific group within it.

b) Covering up evidence of causes of harm to the health of the general public or a specific group within it.

c) Not acting on evidence of causes of harm to the health of the general public or a specific group within it.

d) Opposing treatments which benefit health (not necessarily secretly in this case, although true motivations for doing so would be secret)

e) Using public health interventions as a cover for other activities 
131 Early in the process it was decided to classify the HCTs by subject or plot (i.e. "antivaccination") rather than the effect (i.e. "sterilisation"), while both are equally valid approaches it was decided to classify by subject due to it being easier to find HCTs online by subject rather than by effect.

Through the index fourteen key HCTs were identified, with several having multiple subcategories. The core 14 were:

1. Disease Origin

2. Illegal Drug Use Promotion

3. Food Contamination

5. Anti-Vaccine

6. Family planning campaigns

7. GMOs

8. Chemtrails

9. Water Contamination

10. Disease Research Scam

Each one is numbered, features a brief description and examples, with the example source and country of origin identified. Motifs are listed in square brackets. The term "They" is occasionally used in the HCT descriptions as a stand in for the various governments, organisations etc. thought to be behind the conspiracy, which may vary from version to version. See example below:

\section{Disease Origin}

Deadly disease was created and spread for malicious purposes. Examples:

1. HIV/AIDS was deliberately created by the US government for the purposes of genocide against ethnic minorities within the USA. USA. 

Victim]

Sub-categories are also listed with examples. See example below:

003 Food Contamination

The indexing also identified five key motifs which frequently appeared across the examples.

If an example includes another HCT type this is referenced in square brackets after the type is mentioned. For example, Example 4 of type 004-2, 'Human Guinea Pigs', is: Appendix B for the synopsis and full HCT Index. 
The primary purpose for collecting, organising and classifying HCTs for an index is to provide a tool for future research. A key aspect of this is identification of specific HCTs as certain types to allow for cross cultural comparison or simply aid in the description of the dissemination of HCTs. The HCT Index can be used as a source of examples for qualitative and quantitative research. To illustrate this we provide details of two pilot studies conducted by the authors using content from the HCT Index.

Study 1. Exposure to and belief in health-related conspiracy theories in the UK

\section{Introduction}

Recent research in the United States found health-related conspiracy theories to be widely known, broadly endorsed and also predictive of health behaviours (Oliver, 2014) and a recent survey of found that vaccine-safety related sentiment is particularly negative in the European region (Larson, et al, 2016). Given the potential consequences of belief in health-related conspiracy theories on health behaviours (Bogart \& Thorburn, 2005; Bogart et al, 2010;

214 Oliver, 2014; Jolley \& Douglas, 2014) it is important to assess the extent to which they are endorsed by the general public. This research examines the rate to which people in the UK are exposed to and believe a wide range of health-related conspiracy theories.

\section{Method}

Five-hundred and fourteen adults living in the UK completed the online survey, with a sample of 301 women and 211 men and ranging in age from 18 to 74 (mean $=37.13$, sd = 12.51). Participants were recruited through the Prolific participant recruitment platform in March 2017 and rewarded $£ 1.00$ for the participation. The sample size gives a margin of error of 4.42 at a $95 \%$ confidence level. Survey results were weighted by age, gender, race/ethnicity, highest qualification and UK region to provide a representative sample of the UK population.

After providing their consent and demographic information, participants were presented with 15 health-related conspiracy theories (HCTs). Six of these were sourced from Oliver and Wood (2014) for the purpose of comparison but, with US-specific details altered and language localised to the UK, the remaining nine were sourced from the HCT Index. 
232 Participants were asked to select if they had heard the HCT before, something similar before,

233 or had never heard it. If they had heard it, or something similar before, they were asked to

234 select where they had heard it from (if remembered). They were also asked to rate the extent

235 to which they agreed with the statement that the presented HCT was "likely to be true" on a

236 Likert scale ranging from 0 (strongly disagree) to 6 (strongly agree). The presentation order

237 of HCTs was randomised to avoid order effects.

\section{Results}

240

241 HCTs about vaccinations causing autism (HCT 005-4), the suppression of natural cures for

242 cancer (HCT 012-1) and mobile phones causing cancer (HCT 012-2) were familiar (heard

243 before or heard similar) to at least half the sample. These three HCTs were also the three

244 most believed to be likely to be true:

Natural cancer cures are supressed

Corporations prevent action on mobile phones causing cancer

The government continue vaccinations despite knowing they cause autism

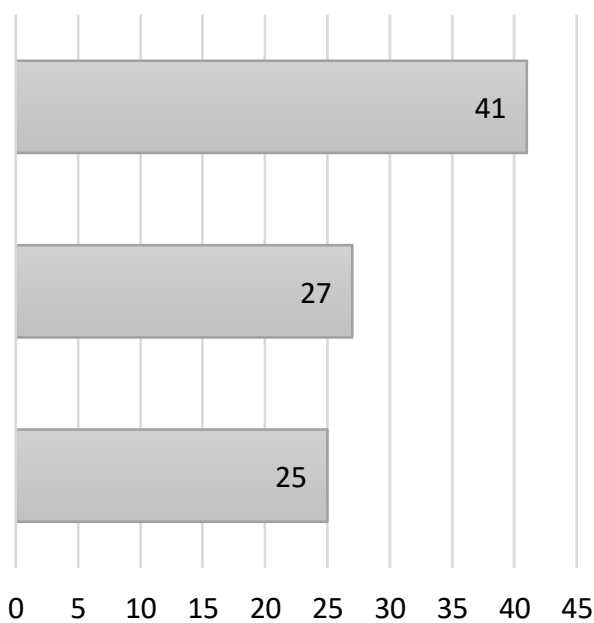

247 Figure 1. Percentage of respondents who agreed with (i.e. gave a score of 4-6) the three most commonly believed HCTs.

HCTs about iodine in table salt being used to limit population growth (HCT 003-1), the United Nations International Children's Emergency Fund (UNICEF) using vaccines as a cover for chemical contraceptive experiments (HCT 005-1-1), genetically modified (GM) foods being used as part of plot to shrink the global population (007-2), fluoridation of water being a method to dispose of chemical waste (009-2-4-1), and the efficacy of the human papilloma virus (HPV) vaccine (005-3) were less well recognised, with less than $25 \%$ of the sample having heard the same or similar before. This could be expected given that some were 
focused on threats to fertility, which appear to be less common in developed countries based on examination of the HCT Index. Three of these were also among those rated as most likely to be false (i.e. received scores 0-2): $85.9 \%$ disagreed with iodine being used to limit population growth; $81.7 \%$ disagreed with water fluoridation being chemical waste; $85 \%$ disagreed with UNICEF using vaccines for contraceptive experiments. In addition to these HCTs about vaccines being used to inject people with tracking microchips (HCT 005-5) and water fluoridation being used to reduce resistance to control (HCT 009-2-1) were among the least believed to be true, being disagreed with by $92 \%$ and $87 \%$ of the sample respectively. In sum, $97 \%$ of Britons are familiar (having heard the same or similar before) with at least one HCT and $78 \%$ are familiar with three or more. $49 \%$ of Britons believed that at least one HCT was likely to be true, while $24 \%$ believed that three or more were likely to be true. See Appendix $\mathrm{C}$ for a table listing the proportions of Britons who reported having heard of the same or similar to the $15 \mathrm{HCTs}$, the most common source of hearing about it and the extent to which they believed it was likely to be true.

\section{Comparison to USA}

The results of this UK-based survey are very similar to those found with a US sample by Oliver and Wood (2014). The same proportion of both samples agreed with at least one HCT (49\%), while the number who agreed with three or more was higher in the UK sample (however it is worth noting that the UK sample were presented with more options). Generally the UK sample were both more likely to believe a HCT was true, and believe it was false, with the undecided option receiving fewer selections than the equivalent in Oliver and Wood (ibid). This could suggest that Britons are firmer in their position on HCTs than Americans, whether in agreement or disagreement, however, it could also be an artefact of the study design (a 0-6 Likert scale rather than three options). In both samples HCTs about vaccinations causing autism, the suppression of natural cures for cancer and mobile phones causing cancer were both the most familiar and the most believed. In general the results suggest that people in the UK and USA share similar attitudes with regards to HCTs. This is despite having different healthcare systems (primarily private sector in the USA and primarily public sector in the UK) and markedly different levels of trust in healthcare professionals: $76 \%$ of Britons believe doctors can be trusted compared to $58 \%$ of Americans (ISSP, 2013). A possible explanation for the shared attitudes is shared exposure to increasingly international forms of media, such as online social media and websites. 
294 Table 1 shows, of the respondents who had heard a HCT before and could remember where

295 they had heard it from, the percentage each source was reported. Forty-three percent of

296 respondents who were familiar with a HCT and could remember the where they heard it

297 reported hearing it from multiple sources. When a single source was reported, online media

298 (websites, social media) accounted for the largest proportion with 46\% stating that they had heard the HCT from a non-news website, a news website, or social media. "Traditional" media, such as television and newspapers was cited as a source for less than $2 \%$ of the HCTs. Word of mouth was a sole source for $9 \%$ of HCTs.

Table 1. Sources for receiving health-related conspiracy theories (HCTs) and the percentage they were cited as a source of hearing about a HCT.

\begin{tabular}{ll}
\hline Source & Percent reported \\
\hline Multiple sources & 42.72 \\
Website (not news or current affairs) & 15.97 \\
Online social media & 15.73 \\
News website & 14.08 \\
In person (known) & 7.47 \\
In person (stranger) & 1.41 \\
Newspaper & 1.41 \\
Other source & 0.86 \\
Television & 0.37 \\
\hline
\end{tabular}

306

307 Although people are familiar with more HCTs than they believe, there was a significant

308 positive correlation between the numbers of HCTs a respondent was familiar with and the 309 number they agreed were likely to be true $(r=.263, p<.001)$. The direction of this effect is

310 not something which can be determined from these data, as it may be that increased exposure

311 to HCTs increases the chance that one will believe in their veracity or it could be that

312 believing in the truth of one HCT leads one to seek out exposure to others, or both could be 313 true and act in a cycle of enforcement. 
Demographic effects

Age

318 There was significant variation between age groups in terms of familiarity with HCTs

319 (Kruskal-Wallis, $X^{2}{ }_{4}=15.35, p=.004$ ), with 35-44 year olds being familiar with fewer HCTs on average than 18-34 year olds $(p=.01)$ and, marginally, 55-64 year olds $(p=.046)$. This difference in familiarity did not translate into a significant difference in mean number agreed with, however $\left(X^{2}{ }_{4}=6.23, p>.05\right)$.

\section{Gender}

There were no significant effects of gender on either familiarity $\left(X^{2}{ }_{l}=1.65, p>.05\right)$ or agreement $\left(X^{2}{ }_{1}=1.84, p>.05\right)$.

Differences between racial or ethnic groups were non-significant in terms of HCT familiarity $\left(X^{2}{ }_{4}=9.49, p=0.05\right)$, however, there were significant differences between groups in mean number of HCTs agreed with $\left(X^{2}{ }_{4}=39.96, p<.001\right)$, with Asian/Asian British respondents agreeing with significantly more HCTs than White respondents $(p<.001)$.

Education level (highest qualification)

There was significant effect of education level on familiarity with HCTs $\left(X^{2}{ }_{6}=15.35, p<\right.$ .001 ), with those respondents with no formal qualifications being familiar with more on average than those respondents secondary education (GCSE, O-levels or equivalents) as their highest education level $(p<.001)$. This difference in familiarity did not translate into a significant difference in mean number agreed with, however $\left(X^{2}{ }_{6}=10.60, p>.05\right)$.

\section{Political affiliation}

342 There was a significant effect of political affiliation on familiarity with HCTs $\left(X^{2}{ }_{2}=16.44, p\right.$

$343<.001)$, with those respondents identifying as politically right being familiar with fewer

344 HCTs on average than those respondents identifying as politically centre $(p=.006)$ or politically left $(p<.001)$. There was also a significant effect of political affiliation on the mean number of HCT's agreed likely to be true, with those respondents identifying as 
politically left agreeing with fewer on average than those respondents identifying as politically centre $(p<.005)$ or politically right $(p<.001)$.

\section{Brexit voting decision}

There was no significant effect of reported Brexit voting decision on familiarity with HCTs $\left(X^{2}{ }_{2}=4.11, p>.05\right)$. There was however an effect on the average number of HCTs believed to be likely to be true $\left(X^{2}{ }_{2}=49.05, p<.001\right)$, with respondents who reported voting for the UK to leave the European Union agreeing with more HCT's on average than respondents who reported voting to remain $(p<.001)$ and those who reported as being eligible to vote but chose not to $(p<.001)$.

\section{Discussion}

In general the results of the survey suggest that both familiarity with and belief in HCTs is relatively high among people in the UK, with almost all respondents being familiar with at least one HCT and half believing that at least one was likely to be true. The results bare striking similarity to the results of a similar survey conducted in the USA with very similar levels of belief and essentially the same HCTs serving as the most familiar and most believed. A possible explanation of this similarity is increasingly shared media between the USA and UK, particularly online forms of media, as these were shown to be the most common source of learning about an HCT. This finding also suggests that belief in HCTs is apparently independent from several distinctive traits, such as the nature of healthcare provision, or post-colonial status. To what extent this would also be true of countries outside of the USA and UK should be examined in further research. A recent global survey on vaccine confidence found significant cross-cultural variation in attitudes towards the efficacy and safety of vaccines (Larson et al 2016), which may suggest similar variation in belief in HCTs.

Despite the result that familiarity with HCT's and agreement that they were likely to be true was positively correlated only one demographic category, political affiliation, was found to effect both familiarity and belief, and this was not in the direction predicted by the correlation. Despite being familiar with fewer HCTs than people who identified as left wing or centre, respondents who identified as right wing believed that more HCTs were likely to be true. Other demographic measures which effected familiarity (age and education) did not 
influence belief, while other measures did not effect familiarity but were associated with belief (race/ethnicity, Brexit voting decision, attitude toward vaccination). The finding that British Asians were more likely to endorse HCTs echoes similar results found in ethnic minorities (African Americans and Latin Americans) in the USA (see Ross, Essien \& Torres, 2006) and could be influenced by similar experiences of racism and a generalised mistrust in state or globalist (in the case of pharmaceutical corporations) institutions, however, such conclusions cannot be reached from the present data and further research would be needed to examine the socio-cultural determinants of increased belief in HCTs among this group. The perception of Brexit leave-voters being 'anti-expert' and 'anti-globalist' could also see support in the result that they were more likely to endorse HCTs, but again, further research is needed to examine the factors involved. The finding that anti-vaccination respondents believed in more HCTs, even when vaccine-related HCTs were excluded suggests a general association between belief in HCTs and belief in vaccine safety. Once again the direction of this association cannot be determined from this data. It may be that general belief in HCTs is reflected in attitudes towards vaccines, or that distrust in vaccines leads to a general distrust of medical institutions and practices.

That people were familiar with more HCTs than they believe demonstrates that there is some form of selection occurring in terms of which are believed. It is clearly not the case that some individuals simply believe whatever HCT they encounter. Future research should examine what makes some HCTs more likely to be believed than others, and how these traits may interact with induvial variation in their believers.

That the level of familiarity and belief is so high in the UK could have serious implications for national health, as belief in HCTs has been shown to have effects on vaccination intentions (Jolley \& Douglas, 2015), adherence to antiretroviral treatment among people with HIV (Bogart et al, 2010), attitudes towards condom-use (Bogart \& Thorburn, 2005), and general avoidance of mainstream medicine (Oliver \& Wood, 2014). The finding that $25 \%$ agree that the government continue to vaccinate children despite knowing that vaccines cause autism and other disorders is of particular concern, as herd immunity exists when a high proportion of people in the population are vaccinated (for example, 19 out of every 20 people need to be vaccinated against measles to protect people who are not vaccinated). Current vaccine coverage, however, is generally high, over $90 \%$, but varies from region to region and 
414 is largely below the $95 \%$ required from herd immunity, so further research is needed to examine how belief in HCTs in the UK influences health behaviour.

\section{Study 2. Focus group on HCTs in rural Sri Lanka}

\section{Introduction}

420

In Sri Lanka, a long history of political commitment to investment in health has led to the development of an extremely high quality national health system in terms of both in- and outpatient care, and excellent performance on population indicators, and has become an example for emulation across the developing world (e.g. Gupta, Dalpatadu, Shanmugarajah, \& Herath, 2013; Samaratunge \& Nyland, 2006). In terms of organisational and fiscal efficiencies, the Sri Lankan national health service (NHS) is said to out-perform the local private health sector and national health systems of other countries of similar socioeconomic development (Hsiao \& Li 2000). However, as in other countries HCTs can threaten people's trust in health interventions and generate concerns about health in general. While theories regarding the nefarious or devious activities of unknown 'others' - Tamils, Muslims, evangelicals, the Indian secret service, the CIA, multinational corporations - are widespread in mainstream (Sinhala Buddhist) Sri Lankan society (Seneviratne 1999), they do not exist self-consciously as 'conspiracy theories' as such (in the sense that those who believe them do not call themselves 'conspiracy theorists' but rather, perhaps, 'nationalists'). Crucially therefore, the research will allow us to explore the extent to which the concept of 'conspiracy theory' itself exists in Sri Lankan culture, with a focus on HCTs.

As part of an investigation into HCT concepts and attitudes we conducted a focus group in which medical interventions, the conspiracy theories related to these and other related issues are discussed.

\section{Method}

444 The research took place in a village in the north-central province of Sri Lanka consisting of 130 households of paddy farmers and their families. Widger has conducted village

446 ethnography at this village since November 2015, with a central focus on health problems associated with agrochemicals and also health conspiracies that surround agrochemicals. 
Participants were recruited through opportunity sampling. There were three females and three males, no ages were recorded. Three researchers were present during the focus group, one of whom acted as a translator. Participants took part in open discussion on the topic of medical and health-related rumours and their attitudes towards them. Topics for discussion were drawn from the HCT Index and contemporaneous ethnographic fieldwork in the locale. The focus group was recruited through opportunity sampling based on Widger's experience of running focus groups in the same community previously.

\section{Results and discussion}

During the focus group a number of HCTs were discussed. In general there were concerns expressed that Muslims are attempting to secretly sterilise the mainstream Sinhala population. Specific examples of these HCTs included a concern that rice provided as aid to the village by Pakistan contained "a certain germ" which would make people infertile (HCT 003-1) or that certain seat covers for motorcycles produced in Pakistan contain a toxic material which sterilises the rider. The sterilising motorcycle seat covers have no direct correlate example in the HCT Index, it would come under HCT 003, contamination, and most resembles HCT 003-1, but is a rare example of contamination of a non-food item. There was also a HCT being spread via social media at the time the researchers were present in the village which suggested that ISIS were posing as medical professionals taking blood samples and secretly injecting people with HIV. The ISIS injecting people with HIV example also has no direct correlate in the HCT Index. It could be considered a variant of HCT 005-2, Disease Spread, but features the taking of blood samples rather than vaccination. Based on discussion within the focus group it is plausible that these concerns are, at least partly, based on observations of reduced fertility compared to the past, with people recognising that younger people are having less children than older generations. While this is caused by changes in lifestyle choices as Sri Lanka experiences the demographic transition, and this is understood by people in Sri Lanka, the observed changes in society may inspire or help propagate fertility related HCTs. These HCTs are also likely influenced by an increase in the Sri Lankan Muslim population, as such Sri Lankan Muslims (or Muslim majority countries) are identified as the conspirators.

480 Generally individuals did not hold an absolute belief in the veracity of these HCTs but expressed being "afraid" or "confused" by not feeling that they are able to determine if the 
rumours they hear are true or false. They felt that the systems in the village were not developed to deal with rumours with origins from outside of the village and as such they cannot determine their veracity. Participants suggested that the government should help determine whether health-related rumours are true, however, they also recognised that people would not necessarily believe statements from authorities due to corruption and the potential for bribery.

Individuals also gave indications about the nature of the natural transmission of health information, HCTs and health rumours. They named a number of sources for this kind of information, such as friends, family and the media, including social media. They also stated that rumours were transmitted where villagers gathered together, such as the market or bathing places. They also suggested that negative information was more likely to be transmitted than positive information with the exception perhaps of personal health information, as it was suggested that individuals are more likely to discuss the illnesses of others than themselves.

\section{General Discussion}

The HCT Index itself can provide a number of interesting insights into the international dissemination of HCTs. For example, from examining the motifs examples we can see that HCTs related to fertility are common (the Fertility Threat motif), largely involving secret attempts to sterilise a population through medical interventions. A very common version is that vaccination programmes are being used to secretly sterilise people. These sterilisation HCTs are almost exclusively found in developing nations, while Western nations seem to be more concerned with survival, with vaccinations being used to spread diseases rather than sterilise. However, while threats of secret sterilisation seem to be largely absent in Western nations, HCTs with children as the victim are common (the Child Victim motif), perhaps reflecting similar concerns. Also, fertility-threat HCTs are found within minority communities in Western nations, primarily the African American community in the examples currently listed (Minority Victim motif). Beyond threats to fertility, by examining type 005, Anti-Vaccination, we see that vaccinations feature in a wide range of HCTs, with wide ranging motives and effects. These include spreading disease, covert genocide and the implantation of tracking microchips. This simple examination of types and motifs provides a number of potential avenues for future cross-cultural research into HCTs, and how certain 
516 types of content and threats are likely to be more successful than others in certain contexts.

517 This could also give insights into how these attitudes could be countered. The two study

518 examples also illustrate the value of the index as a source of material for both quantitative

519 and qualitative research. In both studies the HCT Index provided initial examples which

520 could be adapted to different research paradigms and provide insightful results. Study 2 also

521 demonstrates that the typology can be used to categorise novel HCTs that are identified by

522 participants in qualitative research and allow for comparison with known HCTs that may

523 exist in different environments.

525 Currently, the HCT Index is a working document that is intended to go through further

526 expansion of examples and refinement of types and motifs. A limitation of the current version

527 is a focus on English language sources for the examples (and therefore the types). Non-

528 Western examples are largely from secondary sources, such as anthropological research. As

529 such the Index may not be a generalizable to HCTs globally and may be more representative

530 of HCT beliefs in the English-speaking West than elsewhere. This is confounded by the issue

531 mentioned in Study 2, that in many countries, while beliefs which meet our defining criteria

532 exist, they do not exist self-consciously as 'conspiracy theories' and as such can be more

533 challenging to identify. Further, the number of motifs could be expanded by more detailed

534 analysis of the current examples, and perhaps comparison with the ATU system motifs, as

535 contemporary folklore often reflects motifs seen in traditional folklore. Nonetheless the HCT

536 Index provides a useful framework for future cross-cultural research and the typology is likely to encompass a wide range of beliefs and allow for categorisation of these beliefs. 
Bogart, L. M., \& Thorburn, S. (2005). Are HIV/AIDS conspiracy beliefs a barrier to HIV prevention among African Americans? JAIDS Journal of Acquired Immune Deficiency Syndromes, 38(2), 213-218.

Bogart, L. M., Wagner, G., Galvan, F. H., \& Banks, D. (2010). Conspiracy beliefs about HIV are related to antiretroviral treatment nonadherence among African American men with HIV. Journal of acquired immune deficiency syndromes (1999), 53(5), 648.

Bortolini, E., Pagani, L., Crema, E. R., Sarno, S., Barbieri, C., Boattini, A., ... \& Tehrani J.J. (2017). Inferring patterns of folktale diffusion using genomic data. Proceedings of the National Academy of Sciences, 114(34), 9140-9145.

Brunvand, J. H. (1963). A classification for shaggy dog stories. The Journal of American Folklore, 76(299), 42-68.

563

CDC. (2013). National immunization survey, vaccine for children program, and recent measles outbreaks in the U.S. press briefing transcript. http://www.cdc.gov/media/releases/2013/t0912_measles-outbreaks-data.html (accessed 15th November 2018).

Dundes, A. (1997). The motif-index and the tale type index: A critique. Journal of Folklore Research, 195-202.

Das Gupta, M., Dalpatadu, K. C. S., Shanmugarajah, C. K., \& Herath, H. M. S. S. D. (2013). Multisectoral preventive health services in Sri Lanka: lessons for developing countries in providing public goods in health. World Bank Policy Research Working Paper No.

Hsiao, W., \& Li, K. T. (2000). A Preliminary Assessment of Sri Lanka's Health Sector and Steps Forward. Colombo.

ISSP (2013). International Social Survey Programme: Health and Health Care - ISSP 2011. http://www.issp.org/.

Jolley, D., \& Douglas, K. M. (2014). The effects of anti-vaccine conspiracy theories on vaccination intentions. PloS one, 9(2), e89177. 
Larson, H.J., de Figueiredo, A., Xiahong, Z., Schulz, W.S., Verger, P., Johnston, I.G., Cook, A.R. \& Jones, N.S (2016). The state of vaccine confidence 2016: global insights through a 67-country survey. EBioMedicine, 12, 295-301.

Oliver, J. E., \& Wood, T. (2014). Medical conspiracy theories and health behaviors in the United States. JAMA Internal Medicine, 174(5), 817-818.

Ross, M. W., Essien, E. J., \& Torres, I. (2006). Conspiracy beliefs about the origin of HIV/AIDS in four racial/ethnic groups. Journal of acquired immune deficiency syndromes, 41(3), 342.

Roxby, P. (2018). 'Don't be taken in by anti-vaccine myths on social media'. Retrieved November $15^{\text {th }} 2018$, from BBC News, https://www.bbc.co.uk/news/health-45990874

Samaratunge, R., \& Nyland, C. (2006). Globalisation and social protection reforms in Sri Lanka. South Asia: Journal of South Asian Studies, 29(3), 415-434.

Seneviratne H.L. (1999). The Work of Kings: The New Buddhism in Sri Lanka. Chicago: Chicago University Press.

Uther H.J. (2004). The Types of International Folktales: A Classification and Bibliography. Based on the System of Antti Aarne and Stith Thompson. Helsinki: Suomalainen Tiedeakatemia.

WHO (2018). Measles cases hit record high in the European Region. Retrieved November $15^{\text {th }} 2018$ from World Health Organisation Europe, http://www.euro.who.int/en/media-centre/sections/press-releases/2018/measles-caseshit-record-high-in-the-european-region 